\title{
Iron supplementation consumption behavior predictors based on the Health Belief Model among 9th-grade female students of Birjand in 2018
}

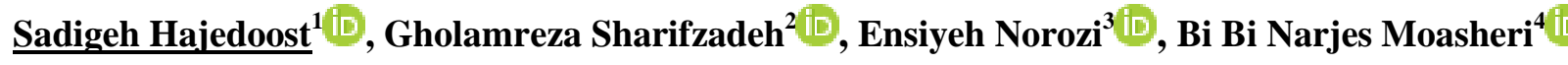 \\ ${ }^{1}$ Student Research Committee, Department of Health education and promotion, Birjand University of Medical Sciences, Birjand, \\ Iran \\ ${ }^{2}$ Social Determinants of Health Research Center, Department of Public Health, Faculty of Health, Birjand University of Medical \\ Sciences, Birjand, Iran. \\ ${ }^{3}$ Department of Health education and promotion, Faculty of Health, Birjand University of Medical Sciences, Birjand, Iran \\ ${ }^{4}$ Corresponding author; Department of Public Health, Faculty of Health, Birjand University of Medical Sciences, Birjand, Iran \\ Tel: +989151636568 \\ Fax: +985631631651 \\ E-mail:n.moasheri@yahoo.com
}

\begin{abstract}
Background and Aim: Anemia caused by iron deficiency, an important health issue is especially in teenager girls. The purpose of this study was, to determine the application of the Health Belief Model (HBM) constructs for the identification of influential factors on iron supplementation consumption among 9 th-grade female students in Birjand.
\end{abstract}

Materials and Methods: This descriptive-analytical cross-sectional study was performed on 517 of 9th grade female students. Students were selected by the randomized multistage sampling method. Data were collected through a researcher-made questionnaire whose validity and reliability was confirmed. The questionnaire included demographic information, HBM structures, and knowledge questions. Data were analyzed by Pearson correlation coefficient and regression model.

Results: The results of the study showed that knowledge of subjects about iron supplementation consumption, was $10.1 \%, 42.6 \%$, and $34.7 \%$, their attitudes are $1.4 \%, 52.6 \%$, and $46 \%$, and also their performance was $57.8 \%, 34.6 \%$ and $7.5 \%$, in poor, moderate and good levels, respectively. Iron supplementation consumption behavior predictors include: Self-efficacy $(\beta=0.196, \mathrm{P}<0.001)$, practice guidelines $(\beta=0.330, \mathrm{P}<0.001)$ and perceived severity $(\beta=0.085, \mathrm{P}<0.046)$. These construct predicted $32.4 \%(\mathrm{R}=0.324)$ of behavioral changes overall.

Conclusion: Students' knowledge and attitude were moderate, and most 9th-grade female students do not take iron tablet, it is suggested that practice, perceived severity, and self-efficacy in students should be strengthened by conducting educational interventions.

Key Words: Attitude; Health Belief Model; Iron; Knowledge; Supplementary; Students 


\section{يشيشبنى كننلههاى روفتار مصرف مكمل آهن بر اساس ملل اعتقاد بهلاشتى در دانش آموزان دختر يايه ذهم

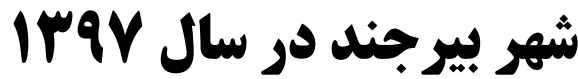

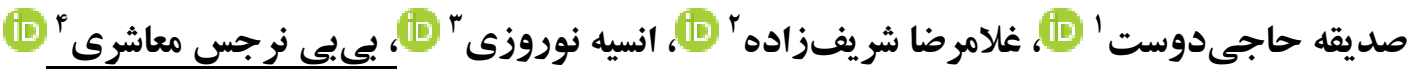

\section{جكيله}

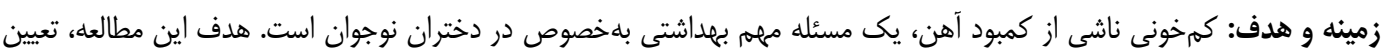

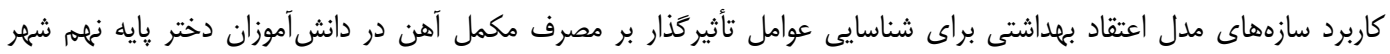

بيرجند بود.

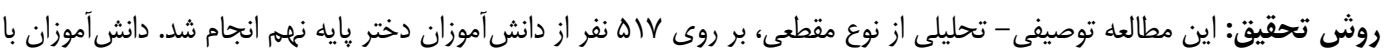

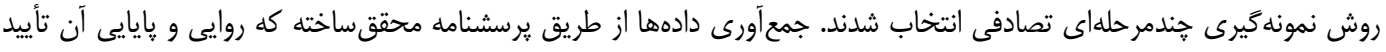

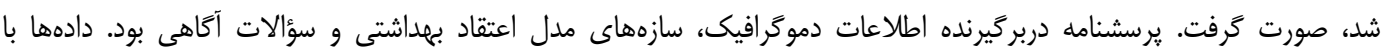

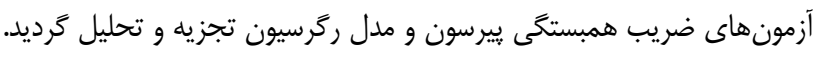

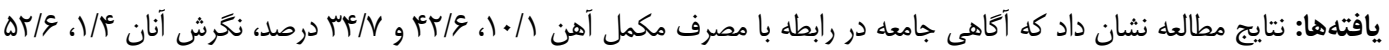

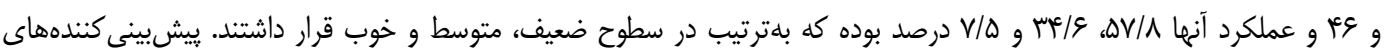

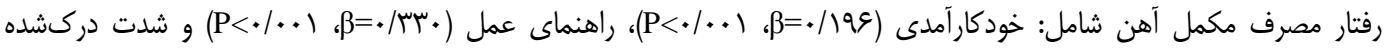

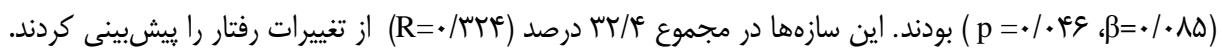

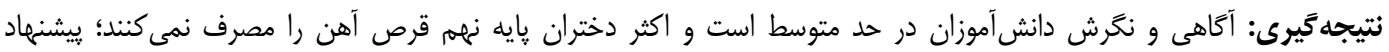

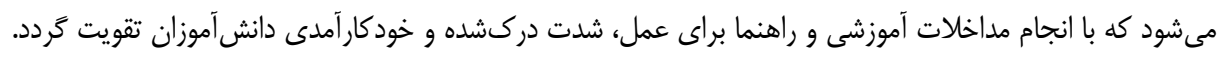
وازههاى كليدى: نكَرش؛ مدل اعتقاد بهداشتى؛ آهن؛ آَاهى؛ مكمل؛ دانش آموزان

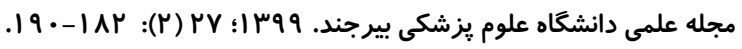
دريافت:

عضو كميته تحقيقات دانشجويى، كروه آموزش بهداشت و ارتقاى سلامت، دانشكاه علوم بزشكى بيرجند، بيرجند، ايران

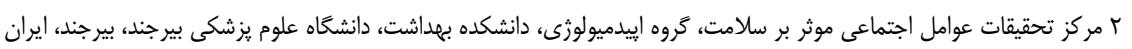

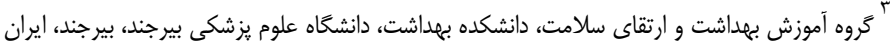

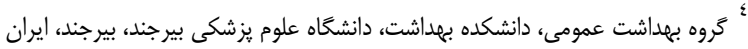

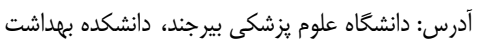


يك قرص فروس سولفات در هفته بلمدات 1 ا هفته توصيه مقدمه

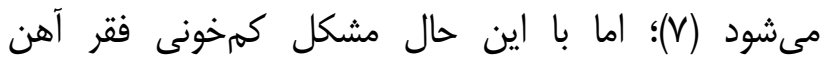

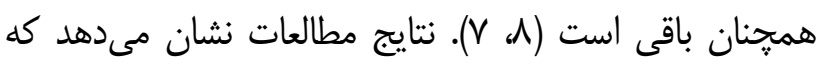

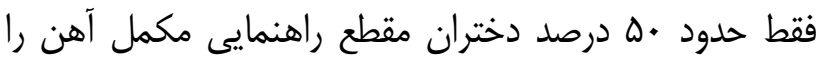

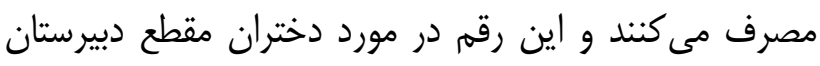

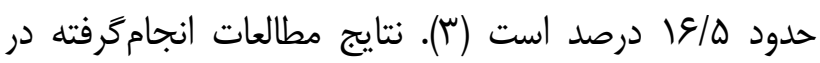
شهرهاى سمنان، تبريز، خابهار، زاهدان، بافت، كرمان و ...

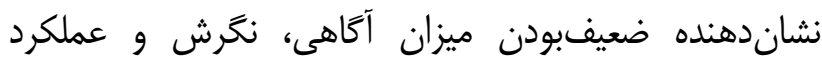

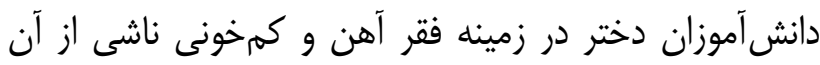

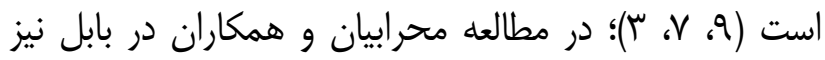

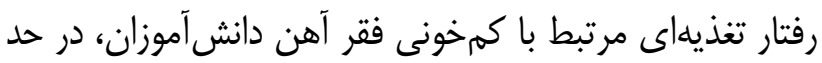

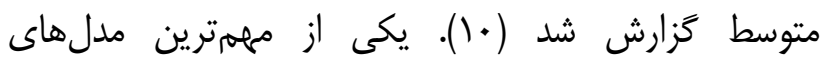
كاربردى در آموزش بهراشت و ارتقاى سلامت، مدل اعتقاد

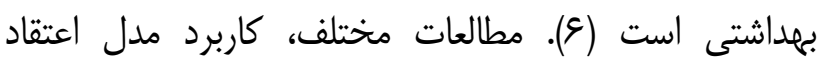

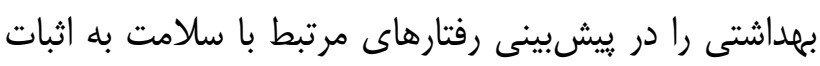

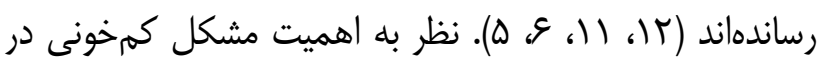

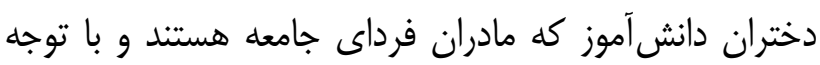
به وضعيت نامطلوب مصرف قرصهاى آهن توسط ماد

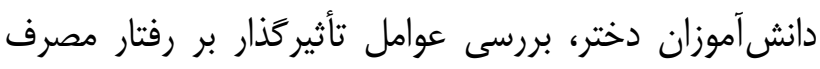

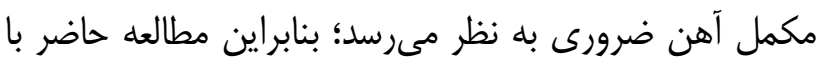

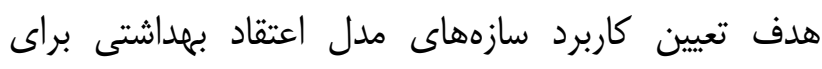

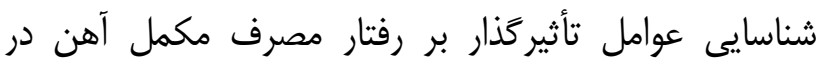
دانشآموزان دختر پايه نهم شهر بيرجند انجام شد.

\section{روش تحقيق}

اين مطالعه توصيفى- تحليلى از نوع مقطعى، در سال

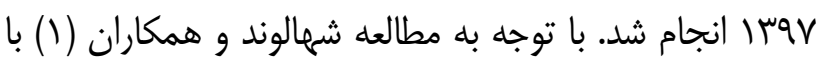

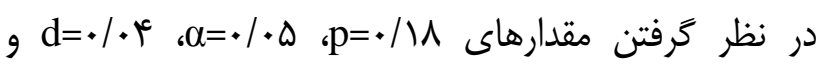

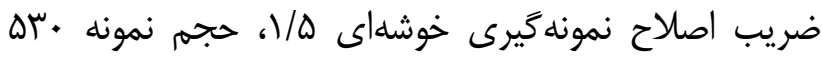

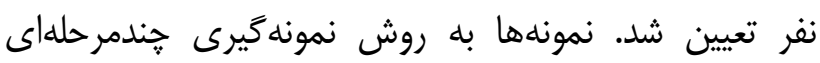

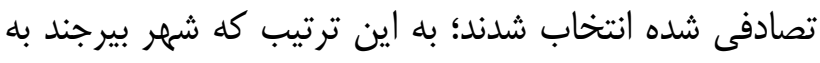

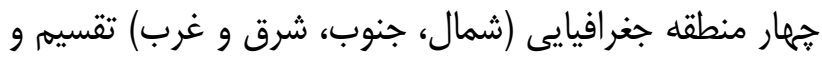

كمبود آهن، شايعترين كمبود تغذيهاى در دنياست و يكى

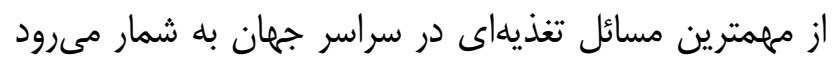

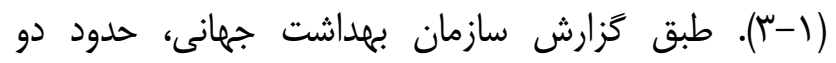

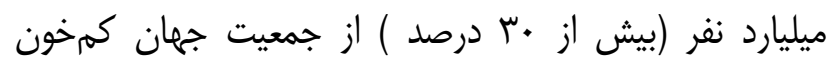

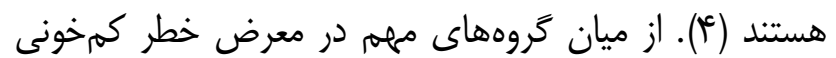

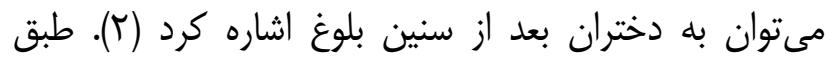

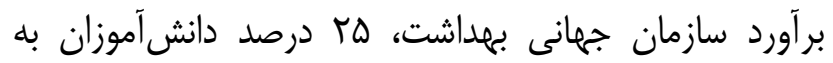

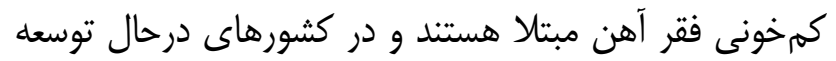

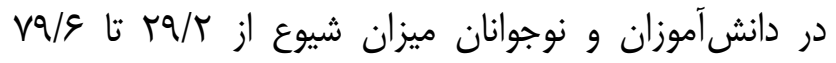
درصد گزارش شده است (ه). شيوع كمخونى فقر آهن در دختران بعد از بلوغ بهدليل

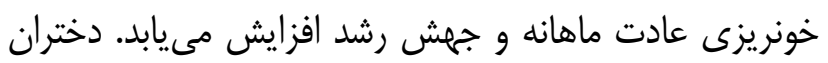

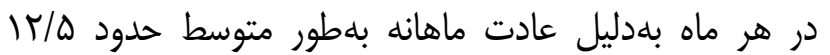

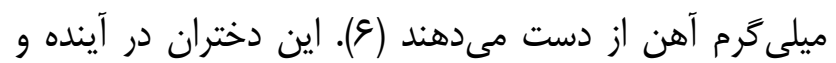

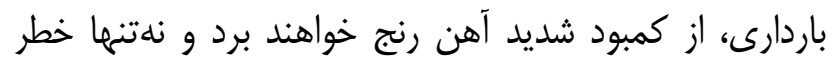

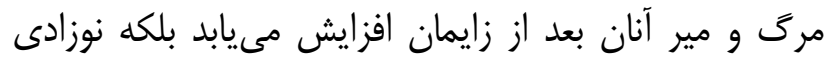
كه به دنيا مى آورند، كموزن يا نارس بوده و ذخاير كافى آهن ندارد (و، عا، ( ). روشهايى مانند بهبود رزيم غذايى، غنىسازى غذاها با

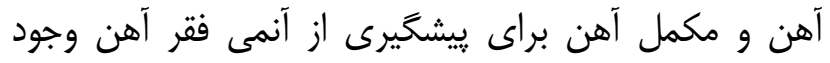

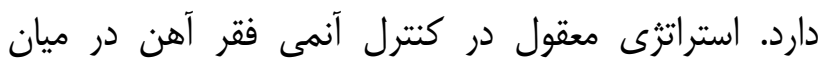

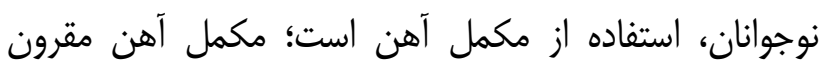

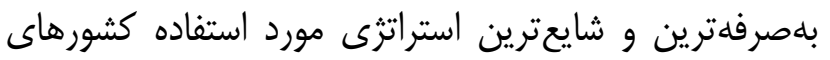

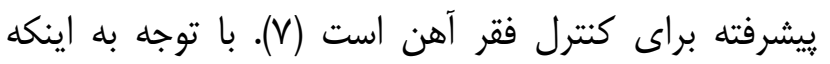

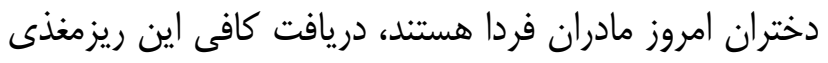

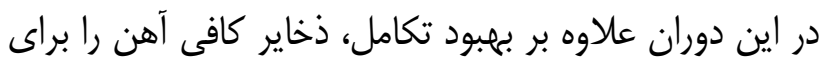

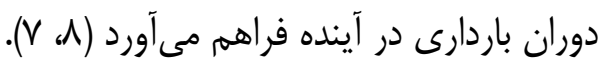

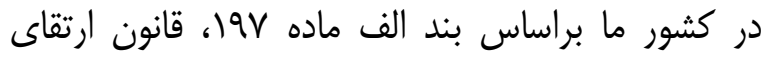

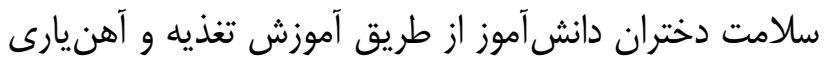

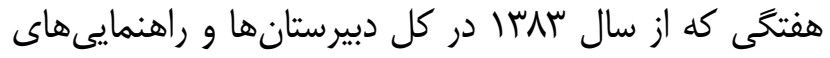

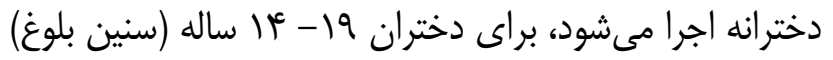


ينجز گزينهاى ليكرت طراحى و از ا تا ه نمره گذارى شد كه در

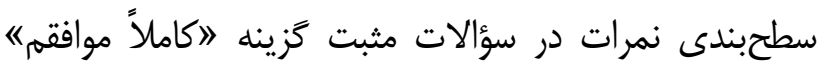

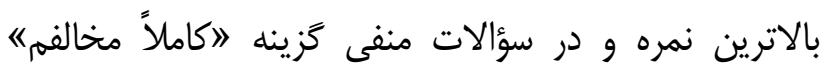

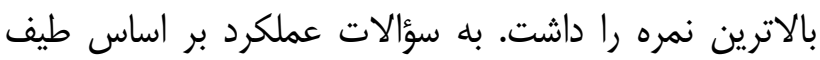

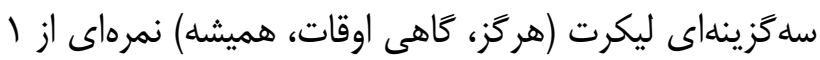

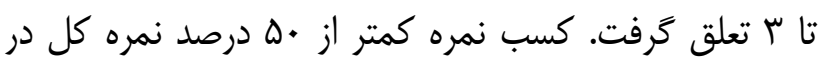

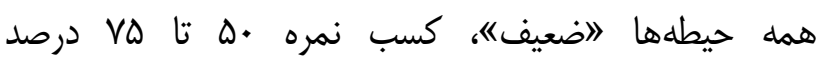

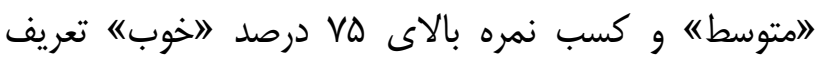
كرديد.

براى تعيين روايى يرسشنامه محققساخته، از روش روايى محتوا و صورى استفاده شد؛ بدين منظور يرسشنامه در اختيار

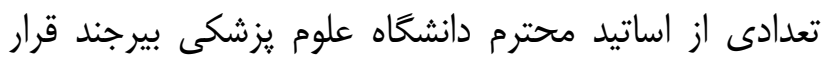

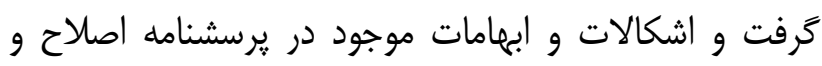

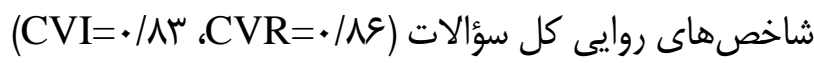

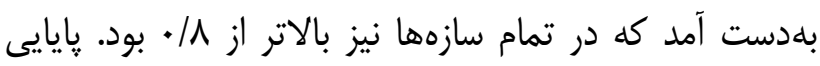

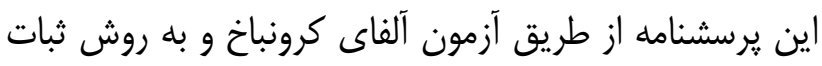

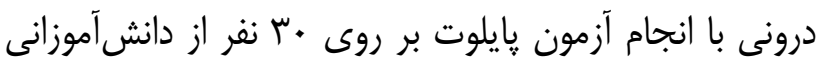

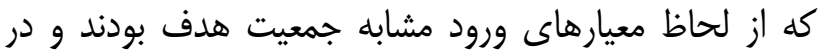

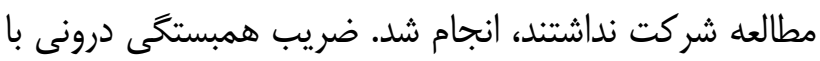

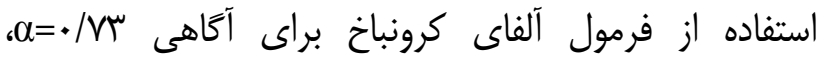

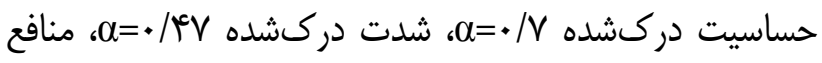
دركشده Q/VT/

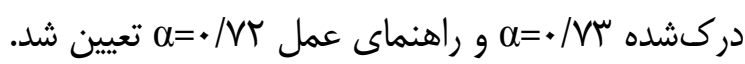

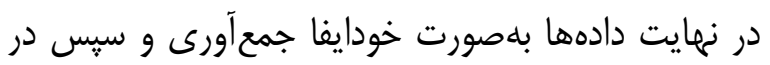

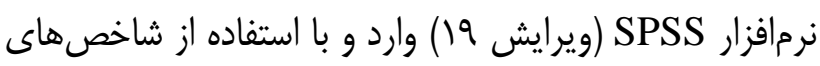

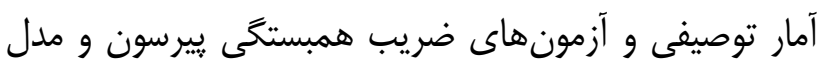

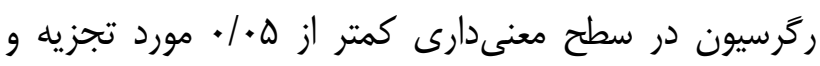

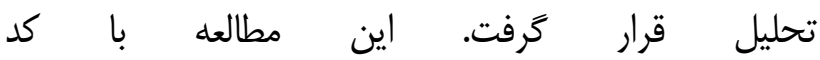
ir.bums.REC.1397.280 در كميته اخلاق دانشگاه علوم يزشكى بيرجند تصويب گرديده و با اعتبارات اين دانشخاه

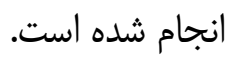

با مراجعه به اداره آموزش و يرورش، اسامى و آدرس مدارس

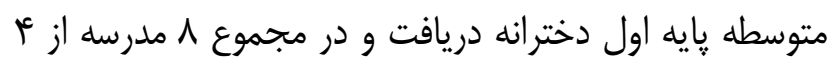

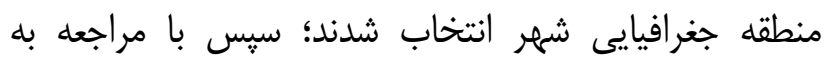

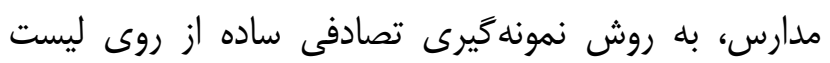
حضور و غياب كلاس نهم، با توجه به معيارهاى ورود شامل:

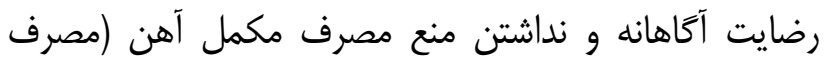

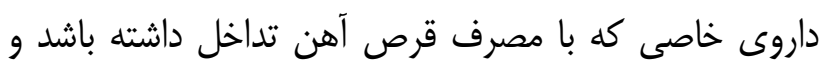

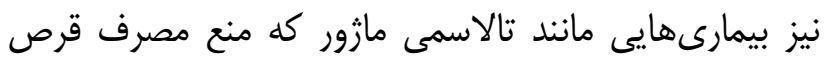

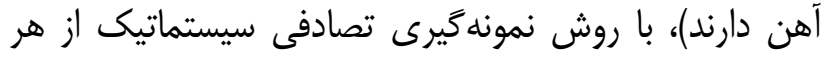

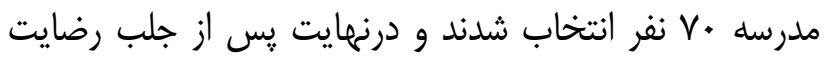

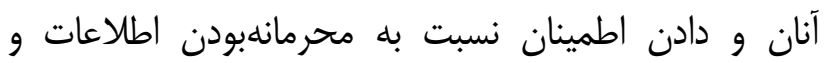
تكميل فرم رضايت توسط آنها، يرسشنامهها تكميل كرديلد.

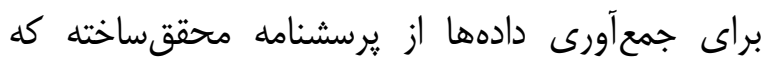

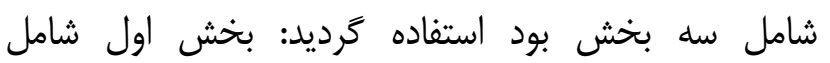

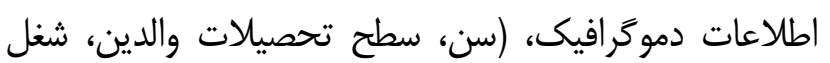

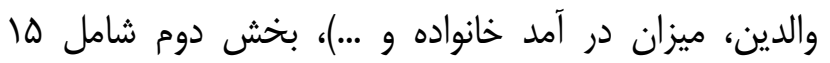

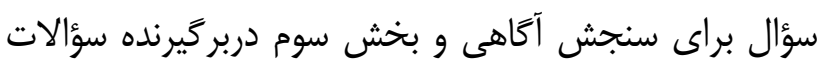
مربوط به سازههاى مدل اعتقاد بهداشتى شامل: حساسيت

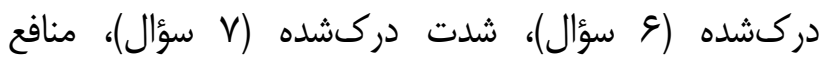

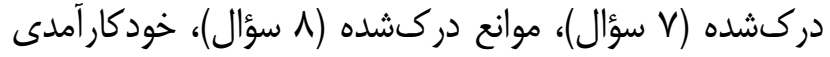

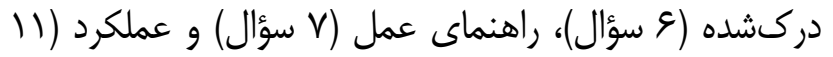

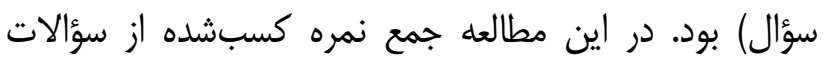

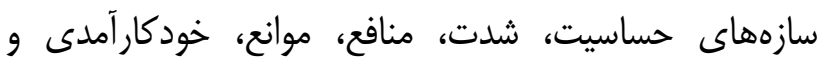

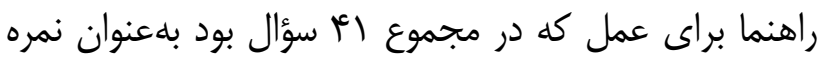
نخرش در نظر گرفته شد.

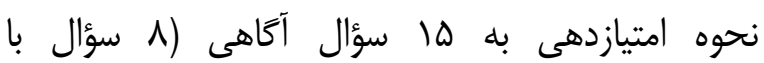

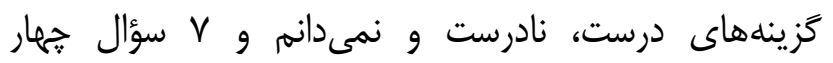
گزينهاى) بدين صورت بود كه به هر سؤال صحيح آَاهى

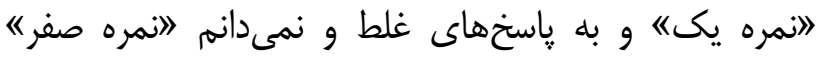

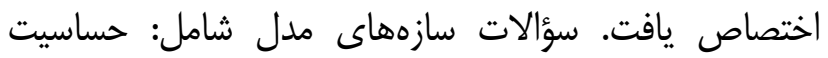

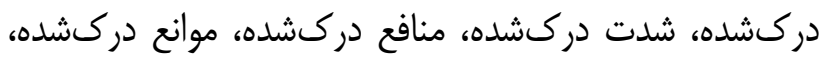

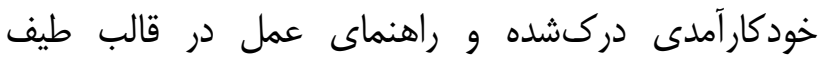


نتايج در رابطه با ميزان آكاهى دانش آموزان نشان داد كه

بافته ها

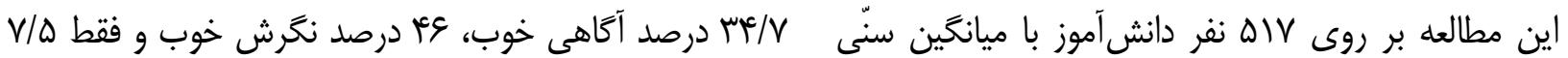

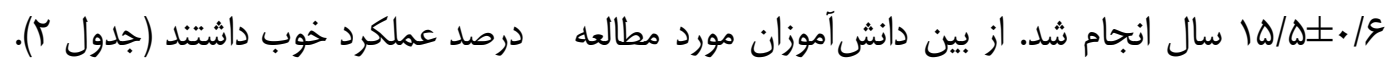

// /

جدول ا - توزيع فراوانى متغيرهاى دموتر افيك در دانشآموزان مورد مطالعه

\begin{tabular}{|c|c|c|}
\hline (درصد) فراوانى & & \\
\hline$r \cdot(r / q)$ & بـسواد & \multirow{4}{*}{ تحصيلات يدر } \\
\hline $1 \& V(\Gamma r / T)$ & ابتدايى - راهنمايى & \\
\hline $\operatorname{llf}(r T)$ & متوسطه & \\
\hline$M \mid G(Y \backslash / \Lambda)$ & دانشخاهى & \\
\hline$T V(\Delta / \tau)$ & بىسواد & \multirow{4}{*}{ تحصيلات مادر } \\
\hline$r \cdot q($ ( $/ / \Lambda)$ & ابتدايى - راهنمايى & \\
\hline$|r|(r M / F)$ & متوسطه & \\
\hline $\mid \varepsilon r(\mu \mid \Delta / \Delta)$ & دانشخاهى & \\
\hline$|F|(r V / T)$ & كمتر از ا ميليون & \multirow{4}{*}{ ميزان درآمد خانواده (تومان) } \\
\hline 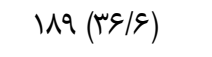 & r-1 ميليون & \\
\hline$\| 1 \%(r / q)$ & r-r-r ميليون & \\
\hline$V^{c}(\mid c / \mu)$ & بيشتر از ؟ ميليون & \\
\hline$V T(1 T / q)$ & ادارى & \multirow{3}{*}{ شغل مادر } \\
\hline rTa (AT) & خانهدار & \\
\hline $19(\mu / 1)$ & مشاغل غير ادارى & \\
\hline $\operatorname{IV}(\Gamma / \Gamma)$ & ا فرزند & \multirow{4}{*}{ ت ت تعداد فرزندان } \\
\hline $1 \cdot V(r \cdot / V)$ & ك فرزند & \\
\hline$M M(\Gamma \& / \mathcal{F})$ & ب فرزند & \\
\hline$r \cdot \Delta(r q / v)$ & $r \leq$ & \\
\hline $198(T r / 1)$ & بلى بل & \multirow{2}{*}{ انجام آزمايش كمخونى } \\
\hline rol (₹V/q) & خير & \\
\hline
\end{tabular}

جدول ؟ - توزيع فراوانى آكاهى، نكَرش و عملكرد دانش آموزان مورد مطالعه در مورد مكمليارى آهن

\begin{tabular}{|c|c|c|c|}
\hline (درصد) فراوانى & (درصد) فراوانى & (درصد) فراوانى & نغير \\
\hline TKE (TY/V) & 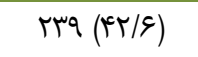 & $\operatorname{ar}(1 \cdot / 1)$ & آكاهى \\
\hline 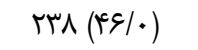 & $T V T(\Delta Y / \mathcal{F})$ & $V(1 / \mathfrak{F})$ & نغخرش \\
\hline чq $(V / \Delta)$ & IVq (re/g) & 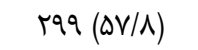 & عملكرد \\
\hline
\end{tabular}


نتايج آناليز رگرسيون خطى جندأنه با استفاده از روش اينتر بلمنظور رييش

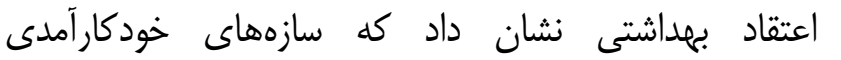

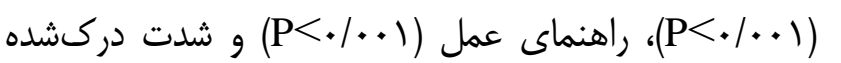

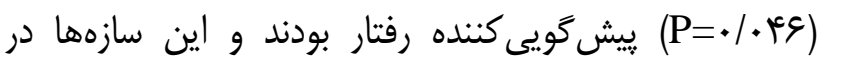

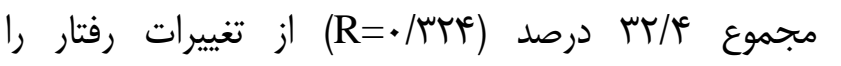

$$
\text { ييش گويى نمودند ( جدول (ا). }
$$

ميانگين نمره حساسيت دركشده در دانشآموزان مورد

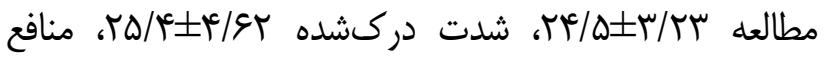
دركشده مطه خودكارآمدى دوكاه /

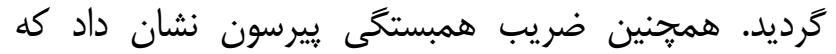

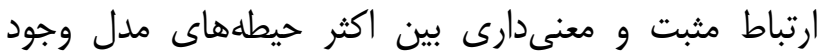
داشت (جدول r).

جدول ب - برآورد ضريب همبستخى بيرسون بين سازههاى مدل اعتقاد بهداشتى در دانش آموزان مورد مطالعه

\begin{tabular}{|c|c|c|c|c|c|c|c|c|}
\hline$\Lambda$ & $v$ & $q$ & $\Delta$ & f & r & $r$ & 1 & متغيّر \\
\hline- & - & - & - & - & - & - & - & آَكاهي \\
\hline- & - & - & - & - & - & - & $\begin{array}{c}\mathrm{r}=. / \mathrm{er} \\
\mathrm{P}<\cdot / \cdots 1\end{array}$ & حساسيت دركشده \\
\hline- & - & - & - & - & - & $\begin{array}{c}\mathrm{r}=\cdot / \Delta \mid \\
\mathrm{P}<\cdot / \cdot \cdot 1\end{array}$ & $\begin{array}{c}r=* / \mu \wedge \\
P<\cdot / \cdots 1\end{array}$ & شدت دركشده \\
\hline- & - & - & - & - & $\begin{array}{l}\mathrm{r}=\cdot / \mu_{q} \\
\mathrm{P}<\cdot / \cdot \cdot 1\end{array}$ & $\begin{array}{c}\mathrm{r}=\bullet / \mu \mathrm{V} \\
\mathrm{P}<\bullet / \cdots 1\end{array}$ & $\begin{array}{c}\mathrm{r}=\cdot / \mathrm{r} \\
\mathrm{P}<\cdot / \cdots 1\end{array}$ & منافع دركشده \\
\hline- & - & - & - & $\begin{array}{c}\mathrm{r}=\cdot / \mathrm{r} \\
\mathrm{P}<\cdot / \cdot \cdot 1\end{array}$ & $\begin{array}{l}\mathrm{r}=\cdot / \cdot \wedge \\
\mathrm{P}=\cdot / \wedge \varsigma\end{array}$ & $\begin{array}{l}\mathrm{r}=. / \cdot 9 \\
\mathrm{P}=\cdot / \cdot r\end{array}$ & $\begin{array}{l}\mathrm{r}=. / 11 \\
\mathrm{P}=. / \cdot 1\end{array}$ & موانع دركشده \\
\hline- & - & - & $\begin{array}{l}\mathrm{r}=-\cdot / \omega^{\circ} \\
\mathrm{P}<\cdot / \cdots 1\end{array}$ & $\begin{array}{c}\mathrm{r}=\cdot / \mathrm{f}^{\mathrm{r}} \\
\mathrm{P}<\cdot / \cdot \cdot 1\end{array}$ & $\begin{array}{c}r=. / 19 \\
\mathrm{P}<\cdot / \cdots 1\end{array}$ & $\begin{array}{c}\mathrm{r}=\cdot / \mu \mathrm{r} \\
\mathrm{P}<\cdot / \cdots l\end{array}$ & $\begin{array}{c}\mathrm{r}=\cdot|r| \\
\mathrm{P}<\cdot / \cdots \mid\end{array}$ & خود كار آمدى \\
\hline- & - & $\begin{array}{c}\mathrm{r}=\cdot / \omega \cdot \\
\mathrm{P}<\cdot / \cdot \cdot 1\end{array}$ & $\begin{array}{l}\mathrm{r}=-\cdot / \mathrm{rV} \\
\mathrm{P}<\cdot / .+1\end{array}$ & $\begin{array}{c}\mathrm{r}=\cdot / \mathbb{} \mathrm{V} \\
\mathrm{P}<\cdot / \cdot \cdot 1\end{array}$ & $\begin{array}{l}\mathrm{r}=-\cdot / \mu 1 \\
\mathrm{P}<\cdot / \cdot \cdot 1\end{array}$ & $\begin{array}{l}\mathrm{r}=-\cdot / \mathrm{r} V \\
\mathrm{P}<+/ . \cdot 1\end{array}$ & $\begin{array}{c}\mathrm{r}=\cdot /{ }^{\mathrm{f}} \\
\mathrm{P}<\cdot / \cdot \cdot 1\end{array}$ & راهنماى عمل \\
\hline- & $\begin{array}{c}\mathrm{r}=\cdot / \Delta T \\
\mathrm{P}<\cdot / \cdot \cdot 1\end{array}$ & $\begin{array}{c}\mathrm{r}=\cdot / \kappa \phi \\
\mathrm{P}<\cdot / \cdots 1\end{array}$ & $\begin{array}{l}\mathrm{r}=-\cdot / \mathrm{r \Delta} \\
\mathrm{P}<\cdot / \cdots 1\end{array}$ & $\begin{array}{c}\mathrm{r}=\cdot / \mu) \\
\mathrm{P}<\cdot / \cdots 1\end{array}$ & $\begin{array}{l}\mathrm{r}=-\cdot / \mathrm{r} \\
\mathrm{P}<\cdot / \cdots 1\end{array}$ & $\begin{array}{l}\mathrm{r}=-\cdot / \mathrm{q} \\
\mathrm{P}<\cdot / \cdots 1\end{array}$ & $\begin{array}{c}\mathrm{r}=\cdot / \mathrm{r} \\
\mathrm{P}<\cdot / \cdots 1\end{array}$ & عملكرد \\
\hline
\end{tabular}

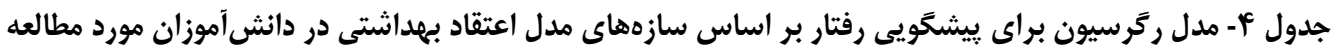

\begin{tabular}{|c|c|c|c|c|c|}
\hline Sig & $\mathbf{t}$ & Beta & Std Error & B & متغير \\
\hline $\mathrm{p}<\cdot 1+\cdots 1$ & $-r / r V m$ & & 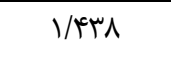 & $-9 / 7 \wedge 9$ & ضريب ثابت \\
\hline $.1 .9 r$ & I/AVT & $\cdot 1 \cdot \mathrm{VV}$ & $\cdot 1 \cdot v^{c}$ & - & آََاهى \\
\hline.$/ 099$ & - /DTE & $-\cdot / \cdot r f$ & س & سس.|•- & حساسيت دركشده \\
\hline $.1 \cdot+8$ & $r / \cdots r$ & .1 .9 & $.1 \cdot$ tet & $\cdot / \cdot \wedge \Delta$ & شدت دركشده \\
\hline$\cdot / \wedge \vee \wedge$ & $-\cdot \mid \backslash \Delta F$ & $-\cdot 1 \cdot \cdot v$ & $\cdot|+4|$ & $-.1 \cdot .9$ & منافع دركشده \\
\hline - Mtip & ./VৎG & سז•|. & . & سז./. & موانع دركشده \\
\hline$\cdot 1+\cdots$ & $p / v^{e c}$ & אTMT & $\cdot|+|+1$ & .1199 & خود كارآمدى \\
\hline $.1 \cdot \cdot 1$ & $V / q \cdot l^{c}$ & $\cdot / r \Delta \Lambda$ & $.1 \cdot$ tr & • & راهنماى عمل \\
\hline
\end{tabular}


عملكرد دانشآموزان ضعيف ارزيابى شده بود. بنابراين نتايج

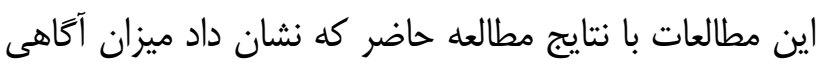

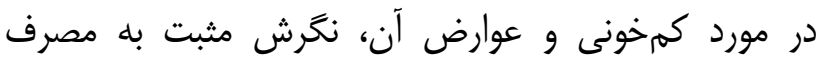

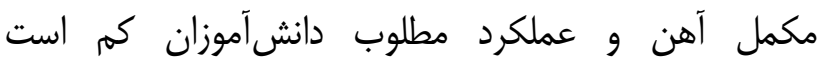

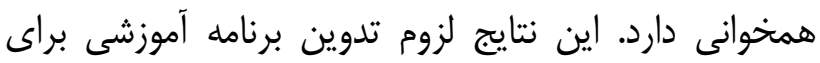

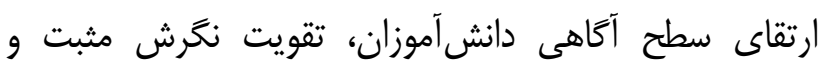

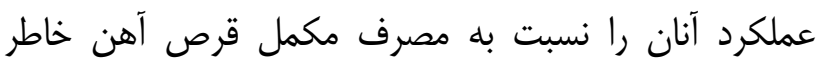

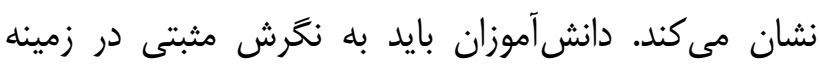

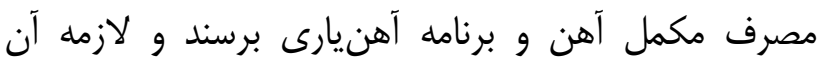

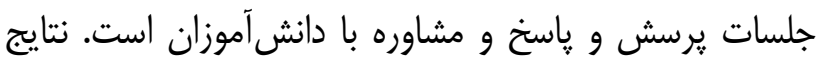

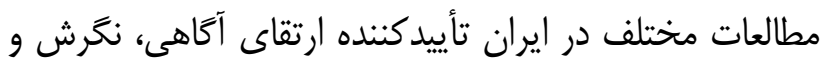

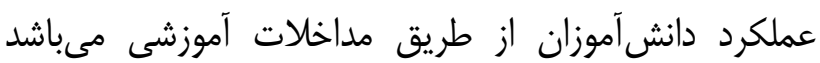

نتايج نشان داد سازمهاى خودكار آمدى، راهنماى عمل و

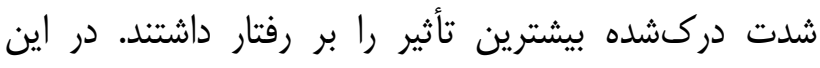

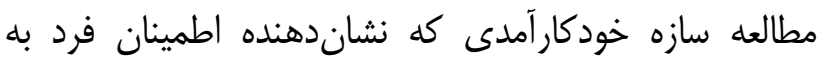
توانايى خود در انجام يك رفتار بهداشتى است ييشكويى كنتلده

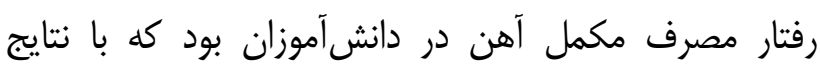

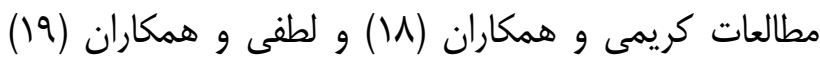

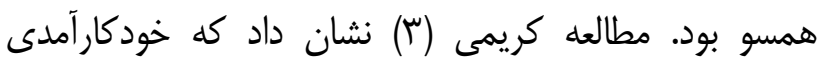

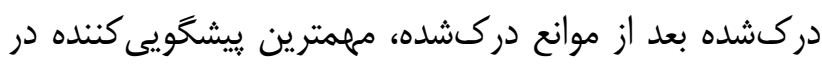

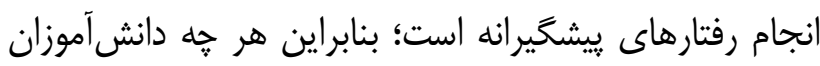

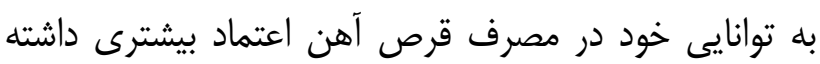
باشند، رفتار مدّ نظر را بيشتر انجام مى دهندا.

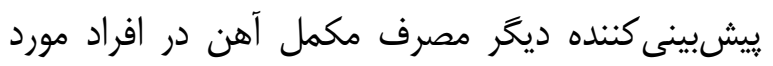

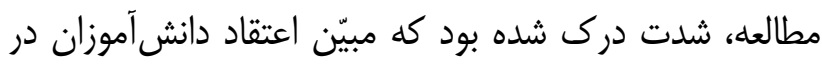

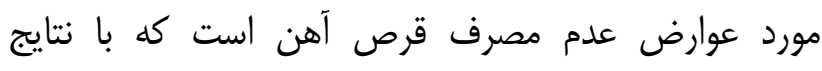

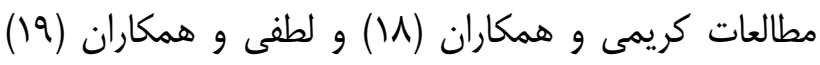

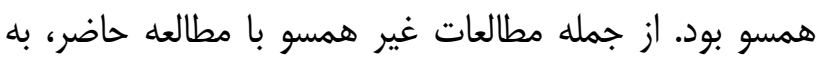

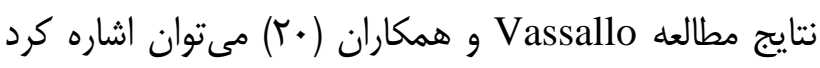

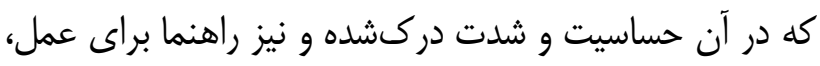
ييشكويى كننده تمايل به مصرف نان غنىشده نبودند؛ اينكه

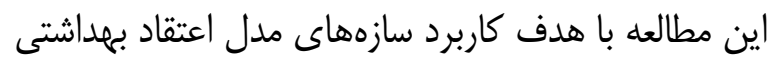

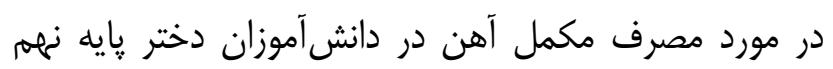

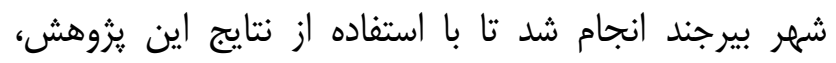

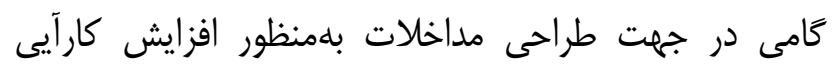

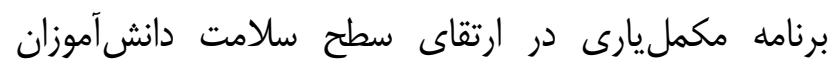

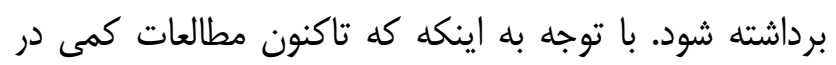

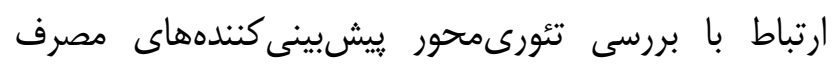

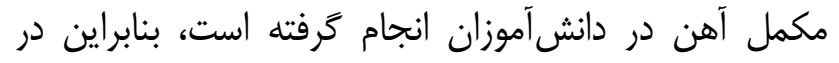
بحث با ساير مطالعات انجامشده مبتنى بر اين تئورى مقايسه صورت خواهد ترفت.

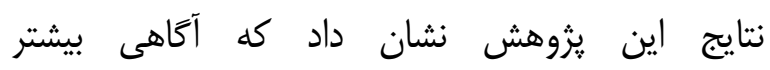

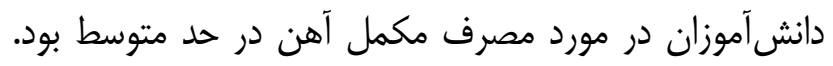

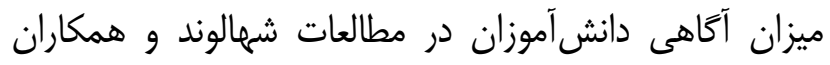
(1)، نقيبى و همكاران (•) (1)، غضنفرى و همكاران (بان (Iان)

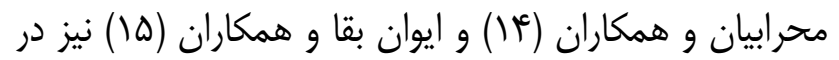
حد متوسط كزارش شد كه با نتايج مطالعه حاضر همخوانى

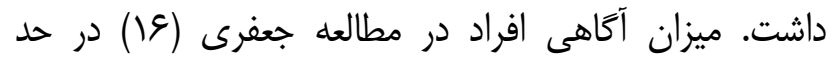
متوسط و ناكافى بود و با انجام مداخله آموزشى، آكًاهى

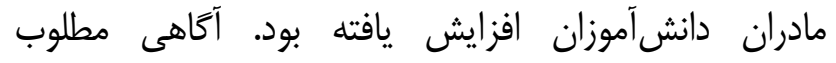

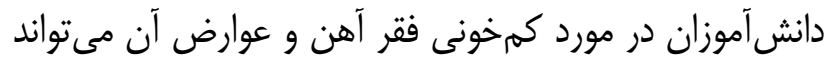

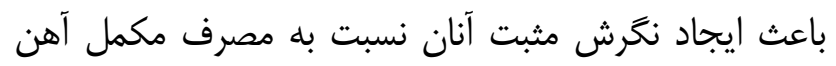

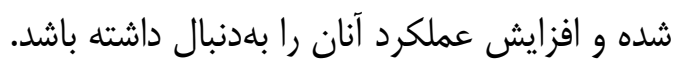

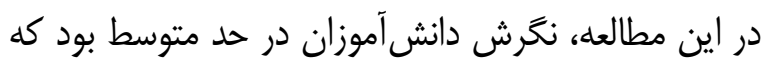

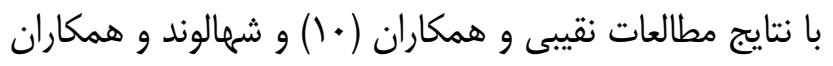

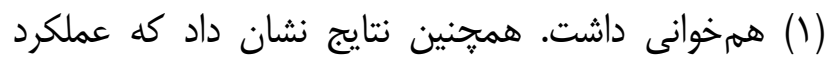

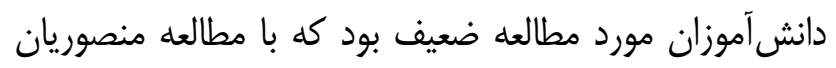
و همكاران (9) همخوانى داشت. در اين زمينه نتايج مطالعات

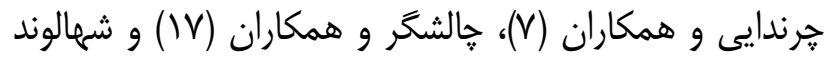

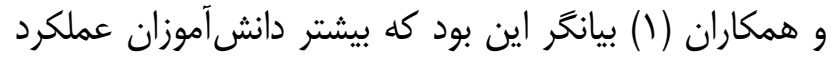

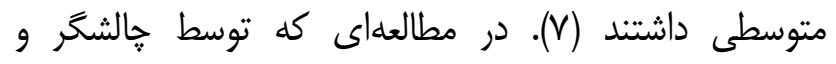

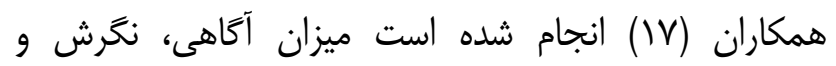


خودكارآمدى و شدت دركشده از اركان اصلى الكوى اعتقاد

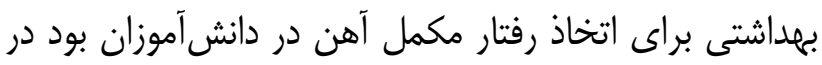

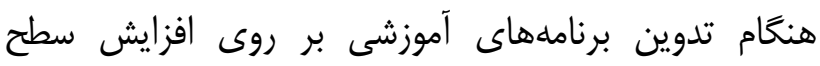

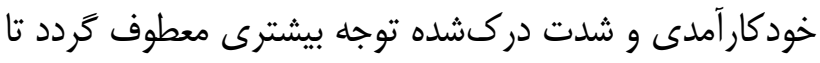

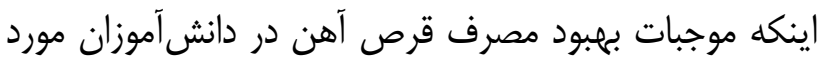

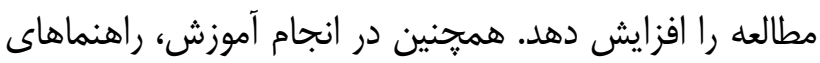
عملى مانند: توزيع يمفلت، كتابِه و .... مى رتواند تقويت كننده باشد.

\section{تقدير و تشكر}

بدينوسيله از معاونت محترم تحقيقات و فنآورى دانشگاه

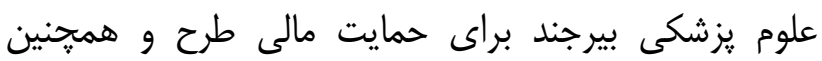

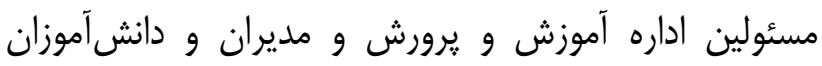

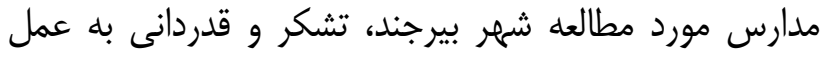

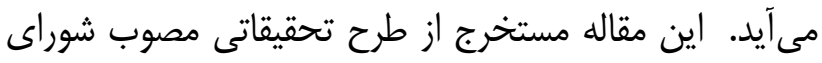

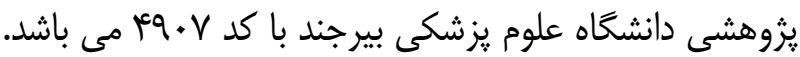

\section{تضاد منافع}

نويسندكان مقاله اعلام مى دارند كه هيج گونه تضاد

$$
\text { منافعى در يزوهش حاضر وجود ندارد. }
$$

افراد خود را در مقابل يك رفتار حساس بدانند يا شدت آن را رآ آنات

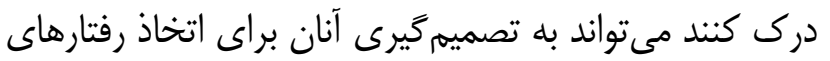

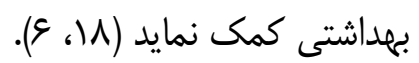
يافتهها در مطالعه حاضر نشان داد سازه راهنما براى

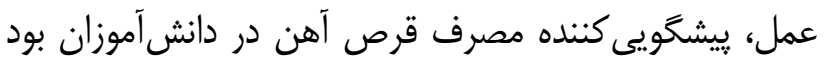

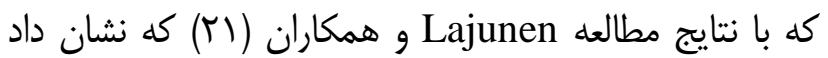
اين سازه ييشگُويى كننده قوى در استفاده از كلاه ايمنى در

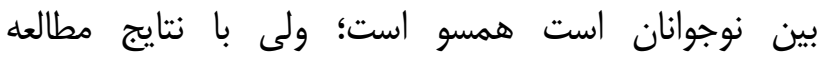
Vassallo همسو نبود.

\section{نتيجه كيرى}

نتايج، بيانگر همبستخى مثبت و معنى معنىار بين آكَاهى و

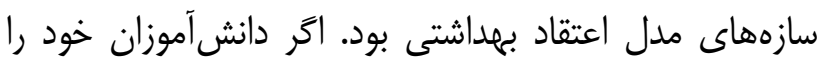
در معرض ابتلا به كمخونى فقر آهن بدانند، موانع انجام رفتار

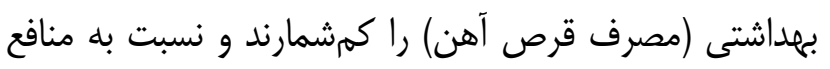
حاصل از مصرف قرص آهن درى بالا و صحيحى ييدا كنند،

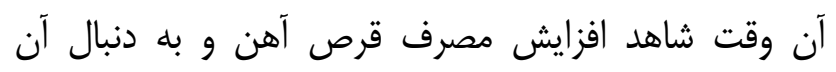

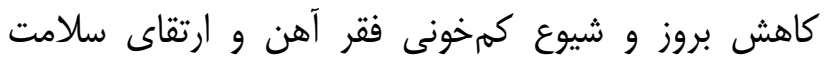

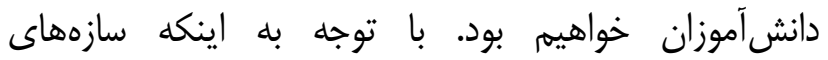

1- Shahalvand F, Ghazanfari Z, Gholami Parizad E, Pournajaf AH, Ranjbar E. Assessing Knowledge Attitude and Practice of Ilam Girl High School Students Towards Iron Deficiency Anemia 2013. J Ilam Univ Med Sci. 2015; 23(4): 209-16. [Persian]

2- Fathizadeh S, Shojaeizadeh D, Mahmoodi M, Garmarodi G, Amirsardari M, Azadbakht M, et al. The Impact of Health Education Based on PRECEDE Model on Knowledge, Attitude and Behavior of Grade Nine Female Students about Iron Deficiency Anemia in Qazvin. J Health. 2016; 7(3): 321-30. [Persian]

3- Karimi B, Hajizadeh Zaker R, Ghorbani R. Intake of Iron supplement and its related factors in jounior and high school girl students of the Iraninan population. Koomesh. 2014; 15(3): 316-24. [Persian]

4- Khani S, Kioumarsi H. Effect of iron deficiency anemia on educational performance and mathematics skills of the third-year guidance school female students in Garmsar 2010-2011. Sci J Iran Blood Transfus Organ. 2014; 11(1): 7786. [Persian]

5- Mirzaei H, Shojaeizadeh D, Tol A, Ghasemi Ghale Ghasemi S, Shirzad M. Application of Health Belief Model (HBM) to Promote Preventive Behaviors Against Iron-deficiency Anemia Among Female Students of High School Fereydan City: A Quasi-Experimental Study. Iran J Health Educ Health Promot. 2018; 5(4): 260-9. [Persian]

6- Mansourian M, Shafieyan Z, Qorbani M, Rahimzadeh Bazraki H, Charkazi A, Asayesh H, et al. Effect of nutritional education based on HBM model on anemia in Golestan girl guidance school students. Iran J Health Educ Health Promot. 2013; 1(2): 51-6. [Persian] 
7- Mohammad Alizadeh Charandaby S, Sehhatieshafaei F, Ebrahimi-Mameghani M, Salmani R. Knowledge and Practice of the students in Tabriz about iron deficiency and iron supplementation. Hormozgan Med J. 2014; 18(3): 26572. [Persian]

8- Noraddin S, Abedini S, Mohseni S. The effect of education on knowledge of high school female's students of Sirik about iron deficiency anemia. Dev Strides Med Educ. 2015; 2(1): 20-5. [Persian]

9- Korourian A, Mirhoseyni M. The prevalence of iron deficiency anemia in high school students of Baft in 2009. Med Lab J. 2014; 7(4): 90-5. [Persian]

10- Naghibi SA, Ahmadi M, Afkhaminia F. Assessment of the Knowledge, Attitude, and Performance of Female Junior High School Students of Neka, Iran, about Iron Deficiency Anemia in 2016. J Health Res Commun. 2018; 4(1): 79-88. [Persian]

11- Ghaderi N, Ahmadpour M, Saniee N, Karimi F, Ghaderi Ch, Mirzaei H. Effect of Education Based on the Health Belief Model (HBM) on Anemia Preventive Behaviors among Iranian Girl Students. Int J Pediatr. 2017; 5(6): 5043-52. doi: 10.22038/ijp.2017.22051.1844

12- Kafaee Z, Noroozi A, Tahmasebi R. Application of Health Belief Model's constructs for predicting regular consumption of folic acid supplements in pregnant women referred to Borazjan's health centers in 2014-15. Iran South Med J. 2016; 19(1): 77-90. [Persian]

13- Shahalvand F, Ghazanfari Z, Gholami Parizad E, Pournajaf AH, Ranjbar E. Assessing Knowledge Attitude and Practice of Ilam Girl High School Students Towards Iron Deficiency Anemia 2013. J Ilam Univ Med Sci. 2015; 23(4): 209-16. [Persian]

14- Mehrabian F, Valipour R, Kasmaei P, Atrkar Roshan Z, Mahdavi Roshan M. Survey Status and Nutritional Behavior to Prevvention of Iron Deficiency Anemia Among High School Girls in Babol City. J Urmia Nurs Midwifery Fac. 2014; 11(12): 1015-23. [Persian]

15- Ivan baga R, Mashoofi M, Hosseini M, Wakili Z, Mahmoodi Keli M, Shahrivar F. The Effect of Education on Knowledge, Attitude \& Practice of Mid-School Girls on Iron-Deficiency Anemia in Khalkhal in 2009. J Health. 2010; 1(3): 57-66. [Persian]

16- Jafari F, Kholdi N, Karimi A. Effectiveness of an educational program on mothers' knowledge about iron deficiency anemia. Koomesh. 2012; 13(4): 419-26. [Persian]

17- Chaleshgar M, Hosseini M, Shojaeizade D, Pishva H. A study of knowledge, attitude and practice of girl students of guidance schools in Ghaemshahr on iron deficiency Anemia. Toloo-e-Behdasht. 2007; 5(1): 1-10. [Persian]

18- Karimy M, Taher M, Azarpira H. Measurement of Health Belief Model Construct in Relation With Nutritional Practices of Pregnant Women in Saveh University of Medical Sciences. Sci J Hamadan Nurs Midwifery Fac. 2016; 24(3): 167-73. [Persian] doi: 10.20286/nmj-24034

19- Lotfi Mainbolagh B, Rakhshani F, Zareban I, Montazerifar F, Alizadeh Sivaki H, Parvizi Z. The effect of peer education based on health belief model on nutrition behaviors in primary school boys. Journal of Research and Health. 2012-2013; 2(2): 214-25. [Persian]

20- Vassallo M, Saba A, Arvola A, Dean M, Messina F, Winkelmann M, et al. Willingness to use functional breads. Applying the Health Belief Model across four European countries. Appetite. 2009; 52(2): 452-60. doi: 10.1016/j.appet.2008.12.008.

21- Lajunen T, Räsänen M. Can social psychological models be used to promote bicycle helmet use among teenagers? A comparison of the Health Belief Model, Theory of Planned Behavior and the Locus of Control. J Safety Res. 2004; 35(1): 115-23. doi: 10.1016/j.jsr.2003.09.020 